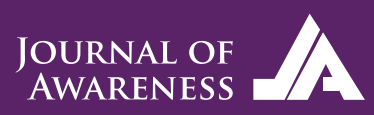

$\left(\begin{array}{l}0 \\ 0\end{array}\right)$
Journal of Awareness

Volume / Cilt: 6, Issue / Sayı: 3, 2021, pp. 123-131

E-ISSN: 2149-6544

https://journals.gen.tr/joa

DOI: https://doi.org/10.26809/joa.6.3.04
Received / Geliș: 04.06.2021

Acccepted / Kabul: 08.07.2021

\title{
Sanal gerçeklik uygulamalarıyla mindfulness temelli stres azaltma programının hemşirelikte kullanımı*
}

\author{
Use of mindfulness applications with virtual reality \\ applications in nursing
}

\begin{abstract}
Gözde Özsezer ${ }^{1} \quad$ (D) Merve Ataç
1 Arş. Gör., Çanakkale Onsekiz Mart Üniversitesi, Sağlık Bilimleri Fakültesi, Hemşirelik Bölümü, Çanakkale, TÜRKIYY, e-mail: gozsezer@comu.edu.tr 2 Öğr. Gör., Çanakkale Onsekiz Mart Üniversitesi, Sağlık Uygulama ve Araştırma Hastanesi, Çanakkale, TÜRKiYE, e-mail: merve.atac@hotmail.com.tr
\end{abstract}

Öz

Sanal gerçeklik (VR) son zamanlarda farkındalığı desteklemek için bir araç olarak kullanılmaya başlanmıştır. Alan yazın incelendiğinde farkındalık pratiklerinin sanal gerçeklik uygulamaları olarak hemşirelik uygulamalarına aktarılmasına yönelik yapılan ulusal ve uluslararası çalışmalara rastlanmamıştır. VR teknolojileri, dikkat becerilerinin deneyimlenebileceği, sürükleyici, ilgi çekici ve kontrollü görsel ve işitsel bir sanal alan sağlanarak çevresel dikkat dağınıklığ ile ilgili zorlukları yararlı bir şekilde ele almaktadır. VR uygulamalarıyla mindfulness temelli stres azaltma programının stres ve kaygının azaltılmasında, dikkati tekrar kazanmada, ileriye dönük belleğin arttırılmasında, anksiyete ve korkunun azaltılmasında, travma sonrası stres bozukluğu, yeme bozukluğu ve ağrı tedavisinde kullanıldığı bildirilmektedir. VR teknolojileri, ruh sağlığı müdahalelerini geniş halk kitleleri için daha erişilebilir hale getirmiştir. Hemşirelik alanında VR mindfulness, yeni bir araştırma alanıdır. Bu nedenle, VR ve diğer dijital uygulamalarda farkındalık ile ilgili süreçleri anlamak için bu konu hakkında daha çok araştırmaya ihtiyaç olduğu görülmektedir. Derlemenin amacı mindfulness stres azaltma programlarının VR ile hemşirelik uygulamaları arasındaki ilişkinin ele alınmasıdır.

Anahtar kelimeler: hemşirelik, farkındalık, mindfulness, sanal gerçeklik

\begin{abstract}
Virtual reality (VR) has recently been used as a tool to support awareness. When the literature is examined, no national or international studies have been found to transfer awareness practices to nursing practices as virtual reality applications. VR technologies beneficially address the challenges of environmental distraction by providing an immersive, engaging and controlled visual and auditory virtual space where attention skills can be experienced. It has been reported that the mindfulness-based stress reduction program with VR applications is used to reduce stress and anxiety, regain attention,
\end{abstract}

Bu çalışma 15-17 Kasım 2020 tarihinde gerçekleştirilen 2. International Conference on Virtual Reality'de sözel bildiri olarak sunulmuştur. 
increase anxiety and fear, and treat post-traumatic stress disorder, eating disorder and pain. VR technologies have made mental health interventions more accessible to the wider public. VR mindfulness is a new research area in the field of nursing. Therefore, it seems that more research is needed on this topic to understand the processes related to awareness in VR and other digital applications. The purpose of this review is to examine the relationship between VR of mindfulness stress reduction programs and nursing practices.

Keywords: nursing, awareness, mindfulness, virtual reality

\section{GİRIŞ̧}

Farkındalık, "şu anda zihni yargılamadan ve anda kalarak bilinçli olarak odaklanma eylemi" olarak tanımlanmıştır (Linehan, 2015). Farkındalık meditasyonunu temel alan müdahaleler, 1979 yilında ilk kez Jon Kabat-Zinn ve arkadaşları tarafından geliştirilmiştir. Mindfulness ise bilinçli farkındalık anlamına gelmektedir. Mindfulness, kişinin şu andaki deneyimlerine merak, açılık ve kabulle kendi kendini kabul etmesini ifade etmektedir (Bishop, 2004). Mindfulness bilinçli bir çaba gerektirmektedir. Özellikle otokontrolü yüksek olan bireylerde kolaylıkla sürdürülebilmektedir (Lutz, vd., 2008; Lymeus, vd., 2017; Lindsay ve Creswell, 2017). Anderson ve arkadaşları (2019) mindfulness uygulaması sırasında ortaya çıkabilecek bir dizi deneyimsel zorluğu belirtmiştir. Bunlar; bilişsel çaba ve hayal k1rıklığı gibi duygusal talepleri, fiziksel çevrenin (örneğin, gürültülü ortamlar ve insanlar) getirdiği görev talepleri, can sıkıntısı, üzücü düşünceler ve duygular gibi olumsuz duygusal veya psikolojik sonuçlardır. Bu zorlukların olasılığını veya etkisini azaltmak için mindfulness uygulamasını uyarlamak, mindfulness temelli müdahalelerin başarısını artırmak için önemli bir husustur. Mindfulness temelli stres azaltma programı da (MBSR) bilinçli farkındalık yaklaşımına dayanmaktadır (Kabat-Zinn, 2011). MBSR, çoğunlukla kronik ağnı şikâyeti yaşayan hastalar üzerinde stresi azaltmanın bir yolu olarak ortaya çıkan bir yaklaşımdır (Segal, vd., 2002a).

Farkındalık uygulamaları, bireylerin deneyimlerine yönelik otomatik düşüncelerine ve uyumsuz duygu durum yönelimlerinin düzenlenmesinde pozitif etkilere sahiptir. MBSR gibi farkındalık uygulamaları, kişilerin olumsuz stres, ağrı ve zihin rahatsızlıkları deneyimleriyle mücadele etmelerine yardımcı olmak için son yıllarda kullanılmaktadır (Choo ve May, 2014). MBSR bu yönüyle de ruh sağlığı açısından yararlı görülmektedir
(Kabat-Zinn, 1990; Aldao, vd., 2010; Blanck, vd., 2018).

Son yıllarda farkındalık pratiğini desteklemek için dijital teknolojinin kullanımı öne çıkmaktadır (Little, 2016; Navarro-Haro, vd., 2017). Örneğin, web tabanlı müdahalelerin farkındalık becerilerini geliştirmek için tasarlanmaya başlanması (Wahbeh ve Oken, 2016; Sevilla-Llewellyn-Jones, vd., 2018) ve farkındalığı destekleyen akıllı telefon uygulamaları bu konuda dikkat çeken gelişmelerdir (Little, 2016; Roquet ve Sas, 2018; Collins, vd., 2019).

MBSR (Kabat-Zinn, 1982) gibi farkındalığa dayalı müdahaleler, genel nüfus tarafından "wellness" programları aracılığıyla hasta olmayanların sağlığını artırmak için kullanılmaktadır (Montero-Marin, vd., 2016). Hasta olmayan popülasyonlarda yapılan araştırmalar, farkındalık uygulamasının, depresif semptomları ve ruminasyonu (Chambers, vd., 2008) azaltmanın yanı sira klinik olmayan popülasyonlarda pozitif duyguyu artırmaya (Erisman ve Roemer, 2010; Jha, vd., 2010) yardımcı olduğunu göstermektedir. Farkındalık uygulaması aynı zamanda bireyin sağlığına yönelik birçok olumlu etki barındırmaktadır. Bunlardan bazıları; bireyin bağışıklığını (Grossman, vd., 2004) ve refah seviyesini yükseltmesi (Linehan, 2015; Campos, vd., 2016) ve dikkatte artış sağlamasıdır (Chambers, vd., 2010).

Farkındalığa dayalı geliştirilen yaklaşımlar kendi içerisinde farklılıklar göstermektedir. Müdahalelerdeki bu farklılıkların, farkındalığın tanımında var olan kavramların çeşitliliğine neden olduğu ileri sürülmektedir (Khoury, vd., 2013). Farkındalık kavramındaki çeşitlilikler; amaca yönelik dikkat geliştirme (Kabat-Zinn, 1990); iç deneyimden kendinden uzaklaşma (Segal, vd., 2002b); kişinin açılılık ve merak farkındalığıyla her bir nesneye bağlanma çabası (Bishop, 2004); ve gerçekliğin ondan kaçınma, ondan kaçma ya da değiştirme girişimleri olmaksızın kabul edilmesi (Hayes, vd., 1999) olarak farklılıklar göstermektedir. Farkındalık kavra- 
mının aynı zamanda öz-şefkat kavramı ile de yakından ilişkili olduğunu belirtmektedir (Baer, vd., 2008; Birnie, vd., 2010). Neff (2003) farkındalığın kendine şefkat için bir ön koşul olduğunu ileri sürerken, Kuyken ve diğerleri (2010), farkındalığa dayalı müdahalelerin başarısının çoğunun öz-şefkatin geliştirilmesine bağlı olduğunu ileri sürmektedir.

Sanal gerçeklik (VR) son zamanlarda farkındalığı desteklemek için bir araç olarak kullanılmaya başlanmıştır (Damen ve van der Spek, 2018; Bruggeman ve Wurster, 2018). VR teknolojileri, dikkat becerilerinin deneyimlenebileceği, sürükleyici, ilgi çekici ve kontrollü (öngörülebilir) görsel ve işitsel bir sanal alan sağlanarak çevresel dikkat dağınıklığı ile ilgili zorlukları yararlı bir şekilde ele alabilir ve dikkati gerçek olandan uzaklaştırabilir (Cikajlo, vd., 2017; Navarro-Haro, vd., 2017; Chandrasiri, vd., 2019). VR, kullanıcıya etkileşimli, görsel-işitsel 360 derece sanal ortamı (VE) yansıtan bir ekrana sahip bir başlığın kullanılması anlamına gelir (Steuer, 1992; Slater, 1999). VR'ler diğer ortamlar (örneğin, bir bilgisayar ekranı) aracılığıyla sunulabilmesine rağmen, VR sistemleri, birden fazla duyuyu uyarmaya yardımcı olan ve bir duyu oluşturabilen daha büyük bir kapsama alanına sahiptir (Witmer ve Singer, 1998; Diemer, 2015). Varlık, kullanıcının bilgisayar tarafından üretilmiş olmasına rağmen ortam gerçekmiş gibi davrandığı bir "gerçeklik yanılsamasına" katkıda bulunur (Slater, 2003; Slater, 2014). Çalışmalar, MBSR programına katılmanın, sağlık çalışanlarının stresini ve tükenmişliğini azaltmak gibi hasta olmayanlar için faydalar sağladığını göstermektedir (Irving, vd., 2009; Montero-Marin, vd., 2015; Kabat-Zinn, vd., 2016). Farkındalığın benzer faydaları farklı kültürlerde de bulunmuştur (Kabat-Zinn vd., 2016).

Farkındalık pratiğinin dijital olarak kullanımına başlanması hem hasta bakımı hem de hemşirelerin iş yüküne olumlu katkısı olacağı düşünülmektedir. Alan yazın incelendiğinde farkındalık pratiklerinin VR uygulamaları olarak hemşirelik uygulamalarına aktarılmasına yönelik yapılan ulusal ve uluslararası çalışmalara rastlanmamıştır. Bu bağlamda, derlemenin amacı mindfulness stres azaltma programlarının sanal gerçeklik ile hemşirelik uygulamaları arasındaki ilişinin ele alınmasıdır.

\section{SANAL GERÇEKLİK UYGULAMALARIYLA MİNDFULNESS TEMELLI STRES AZALTMA PROGRAMININ KULLANIM ALANLARI}

VR sistemleri ve bunların uygulamaları farkındalığa neden olmaya ve insanların farkındalık becerilerini artırmaya yardımcı olmaktadır. Bununla birlikte bu sistemlerin daha iyi bir refah sağladığını gösteren çalışmalar da vardır (Gromala, vd., 2011; Shaw, vd., 2011; Choo ve May, 2014; Crescentini, vd., 2016; Kosunen, vd., 2016; Cikajlo, vd., 2017; Asati ve Miyachi, 2019; Navarro-Haro, vd., 2019; Chandasiri, vd., 2019; Seabrook, vd., 2020; Girardeau, vd., 2020). Örneğin, VR sistemi kullanarak kronik ağrı hastalarında farkındalığı artırmanın kronik ağrıyı azaltmada etkili olduğu bulunmuştur (Gromala, vd., 2011).

Farkındalık uygulamaları bireylerin stresinin azaltmada etkili olduğu yapılan çalışmalarda görülmektedir. VR ile farkındalığın araştırıldığı Shaw ve diğerlerinin (2011) yapmış olduğu bir çalışmada katılımcıların streslerini azaltmada yardımcı olacak eğitim için sürükleyici bir sanal ortam olan Meditasyon Odası tasarlamıştır. Uyarılmayı izlemek için biofeedback sensörleri benimsenmiş ve bu veriler de VE'nin görsel varlıklarını etkilemiştir. Sonuç olarak, katılımcılar, VE'nin sürekli değişen görsel geribildirimini gözlemlerken, kontrol grubunda tek başına biofeedbackden daha etkili olan stres seviyelerini başarıyla yönetmiştir (Shaw, vd., 2011).

Choo ve May (2014), farkındalık meditasyonu sırasında dikkatlerini dağıtmaya çalışmadan, zaman içinde kullanıcının ilgisini korumak amaciyla bir VR uygulaması tasarlamıştır. Katılımcıları dikkatli bir şekilde meditasyon yaptığ 1 için ödüllendirmek için 'Serenity' adlı bir puan sistemi kullanılmaktadır. Katılımcılar topladıkları 'Serenity' ile meditasyon seansı bittikten sonra ödüllendirilmektedir. Kazanılan 'Serenity' miktarı, katılımcı ne kadar kötü veya mükemmel performans sergilediğine bakılmaksızın tamamlanan her dikkatli ders için statik bir miktardır. Önlem olarak içeriklere kilit konmuştur. Bölümler tamamlandıkça sırasıyla kilit açılmaktadır. 'Serenity' ödül sistemine entegre edilmiştir. Farklı meditasyon derslerine sahip yeni farkındalık sahneleri, önceden belirlenmiş miktarlarda 'Serenity' ile açılmaktadır. Bu nedenle, bir kullanıcı yazılımda ne kadar çok farkındalık meditasyonu gerçekleştirirse, daha fazla farkındalık konumu elde etmeye o kadar 
yaklaşmaktadır. Zamanla yeni farkındalık derslerinin kilidini açmak, yeni kullanıcıların başlangıçta çok fazla seçenek arasında kalmasını engellemektedir. Ayrıca zamanla ödül olarak yeni derslerin kilidi açılmaktadır. Her dikkatli oturum sırasında katılımcıların duygusal ilerlemesi Emotiv EEG başlığ1 ile takip edilmektedir. Gerçek zamanlı olarak soyutlanmış görsel geri bildirim sağlamaktadır. Katılımcı gevşedikçe ve nefes alıp vermesi yavaşladıkça, içsel hislerine odaklanmaya başladığında, Emotiv EEG meditasyon puanlarını (sayısal olarak 0 ile 1 arasında temsil edilir, burada 1 olası en yüksek puandır) ve katılımcıların sakinliğini ve diğer duygusal durumlarını takip edebilmektedir (Choo ve May, 2014).

Ayrıca mobil uygulama olan Hiatus Sistemi (VR stres azaltma mobil uygulaması gerçek zamanlı biofeedback kullanarak MBSR için bir öğretim aracı görevi görmektedir. Uygulama hem tedavi sirasinda hem de sonrasında hastaların stresini azaltmak için tasarlanmıştır. Hiatus Sistemi, kullanıcıya yaklaşık 20 dakikalık seanslarla, MBSR meditasyonunu uygulamaya başlarken doğru nefes alma yöntemini öğretmektedir. Deneyim sırasında kullanıcı, uçmak istediği yöne bakarak büyük bir ağacın etrafında yavaşça uçabilmektedir. Kullanıcılar nefesini verdiklerinde, basınç sensörü aracılığıyla iletilen veriler sayesinde sanal dünyada sırtında hafif bir rüzgar hissetmekte ve hızlarını geçici olarak artırmaktadır (Bruggeman ve Wurster, 2018). Crescentini ve diğerleri (2016) tarafından yapılan araştırmada, katılımcılardan 8 haftalık bir farkındalık meditasyon kursu tamamlandıktan sonra stres çağrıştıran VR senaryolarını gözlemlemeleri istendikten sonra, durum farkındalığında bir artış ve stres tepkisinde azalma olduğu belirtilmiştir (Crescentini, vd., 2016).

Cikajlo ve diğerlerinin (2017) VR mindfulness uygulamasının stres ve kaygı azaltma terapisi etkinliğine etkisinin araştırıldığı çalışmasında $(\mathrm{n}=8)$ mindfulness eğitmeni ve 3D VR kulaklıkları olan katılımcılar için bir Web arayüzünden oluşan bulut tabanlı bir sistem geliştirilmiștir. Mindfulness eğitmeni, kulaklık kullanarak katılımcllar ile Web arayüzü üzerinden iletişim kurmaktadır. Web uygulaması ile sanal odalarda, 360 derecelik videolar ve gerçek etkileşimlerle veya bağımsız meditasyonlarla grup oturumları gerçekleştirilmiştir. Mindfulness programı, geliştirilmiş sanal ortamlar için özel olarak 8 haftalık MBSR kursu olarak tasarlanmıştır. Katılımcıların çalışma sırasında daha yüksek yaşam doyumu seviyesine ulaştığı ifade edilmiştir. Ayrıca VR mindfulness uygulamalarını kullanımı kolay ve kullanıcı dostu olduğu ve çeşitli gruplar için uzaktan farkındalık eğitimi için kullanışlı olduğu vurgulanmıştır (Cikajlo, vd., 2017).

Seabrook ve diğerlerinin (2020) gerçekleştirdiği $(n=37)$ kontrollü bir laboratuvar ortamında bir VR mindfulness uygulaması kullandığı pilot çalışmada durum farkındalığındaki büyük ve istatistiksel olarak anlamlı bir artış olduğu, VR uygulamasının farkındalığı desteklemede başarılı olduğu belirtilmektedir (Seabrook, vd., 2020).

Dikkati yeniden kazanmada da VR mindfulness uygulamaları kullanılmaktadır. Asati ve Miyachi (2019)'nin çalışmasında birden fazla hedefi olan bir okçuluk oyunu da dahil olmak üzere, özel olarak rahatlatıcı bir VR ortam oluşturulmuştur. 12 katılımcının dikkat süresi, VR seansindan önce ve sonra bir aksiyon olmayan video oyunu skoru ve Muse kafa bandı EEG sinyalleri ile test edilmiştir. 10 dakikalık VR oturumu katılımcıların deneyimlerine göre puanlarını arttırmıştır. Muse kafa bandı verilerine göre, katılımcıların VR deneyimlerinden bağımsız olarak sakinlik puanları artmıştır. Deneyden sonra, tüm katılımcılar günlük aktivitelerine devam etmek için rahatladıklarını bildirmişlerdir (Asati ve Miyachi, 2019).

Kosunen ve diğerlerinin (2016) çalışmasında sanal gerçekliği neurofeedback ile birleştiren bir nöroadaptif VR meditasyon sistemi olan RelaWorld sunulmaktadır. Başa takılan bir ekran kullanarak, kullanıcılar meditasyon egzersizleri yaparak sanal bir dünyada yükselmektedir. Sistem, EEG yoluyla kullanıcıların beyin aktivitesini gerçek zamanlı olarak ölçmekte ve konsantrasyon ve gevşeme seviyesi için tahminleri hesaplamaktadır. $\mathrm{Bu}$ değerler daha sonra sanal gerçeklikle eşleştirilmektedir. 43 denek üzerinde yapılan çalışmada, RelaWorld sisteminin daha derin bir rahatlama sağladığı belirtilmektedir (Kosunen, vd., 2016).

MBSR temelli VR uygulamaları ileriye dönük bellek için de kullanılmaktadır. Girardeau ve diğerlerinin (2020) çalışmasında, katılımcılardan $(n=80)$ sanal bir kasabada bir yürüyüş sırasında belirli anlarda (zamana dayalı öğeler) veya yerlerde (olay temelli öğeler) eylemleri hatırlamaları istenmiştir. Çalışmada ileriye dönük bellek için önemli bir fark görülmemesine rağmen VR uygulamasının olumlu etkisinin yadsınamayacağ 1 vurgulanmaktadır (Girardeau, vd., 2020). Fakat yapılan bazı araştırmalar bunun tam tersini göstermektedir. Bu 
çalışmalarda, zihinsel sağlık veya durum farkındalığı üzerindeki etkisiyle ilişkili olarak VR destekli farkındalık uygulaması değerlendirilmiştir (Gomez, vd., 2017; Navarro-Haro, vd., 2017; Flores, vd., 2018; Chandasiri, vd., 2019). Klinik olmayan bir popülasyondan uzman meditasyoncularla yapılan bir değerlendirmede, Navarro-Haro ve diğerleri (2017), sanal gerçeklik destekli bir farkındalık uygulamasının durum farkındalığını ön testten son teste kadar artırdığını, üzüntü ve kaygı duygularını azalttığını ve gevşeme duygularını artırdığını göstermiştir. Yine Navarro-Haro ve diğerlerinin (2019) birinci basamakta yaygın anksiyete bozukluğunun (YAB) tedavisine yönelik farkındalık temelli müdahalenin VR davranış terapisi uygulaması olarak ve olma-

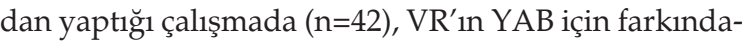
lık temelli yaklaşımlar sunduğu, tedaviye bağlılığ1 ve farkındalık uygulaması için motivasyonu artırmak için iyi bir araç olabileceği ifade edilmektedir (Navarro-Haro, vd., 2019). Chandrasiri ve diğerleri (2019), klinik olmayan, yetişkin örnekleminde $(n=32)$ nefes odaklı bir farkındalık uygulamasıyla eşleştirilen, VR'de sunulan bir plaj sahnesinin çok yönlü bir video kaydını değerlendirmişlerdir. Navarro-Haro ve diğerlerinin (2017) bulgularıyla tutarlı olarak, Chandrasiri ve diğerleri (2019), VR kullandıktan sonra durum farkındalığının önemli ölçüde arttığı bulunmuştur. Bununla birlikte, çalışmaları, VR özelliklerinin farkındalığı nasıl ve neden desteklediğine dair sınırlı bilgi sağlamaktadır.

Diğer girişimler arasında, eğlenceli deneyimlerle farkındalığ 1 geliştirmeyi amaçlayan VR sistemleri (Amores, vd., 2016) ve derin rahatlama, mevcudiyet ve derin meditasyon seviyeleri sağlamak için neurofeedback ile birleştirilen VR sistemleri (Kosunen, vd., 2016) bulunmaktadır.

VR maruziyetinin hem anksiyete bozukluğu olan hem de anksiyete bozukluğu olmayan hastalarda anksiyete ve korku tepkilerinin psikofizyolojik semptomlarını (örneğin elektrodermal aktivite açısından) ortaya çıkaracak ve değiştirecek kadar deneysel gerçekçilik açısından çok etkili olabileceği gösterilmiştir. (Diemer, vd., 2014). VR'de maruz kalma, aslında, anksiyete bozukluklarının tedavisinde ve özellikle, belirli uyaranlara sahip durumlara maruz kalma yoluyla belirli fobilerin tedavisinde etkili olur (Sanal Gerçeklik Maruz Kalma Terapisi). Fobik bozukluklarda sanal gerçekliğin kullanılması, kişiler için imkansız olarak görülen deneyimlerin gerçekleşmesini mümkün kılar (Costa, vd., 2018). Örneğin VR deneyimiyle bir uçaktan atlamak, yavaş yavaş örümcek dolu bir ortamda dalmak (Miloff, vd., 2015), yükseklik korkusu olanlarda balkon korkuluklarına yavaş yavaş yaklaşmak (Rothbaum, vd., 1999) olabilir. Dahası, sanal dünyaya dalma, düşük hayal gücüne sahip veya in vivo maruziyeti reddeden denekler için de yararlıdır (Costa, vd., 2018).

VR ayrica travma sonrası stres bozukluğunun tedavisinde de kullanılmaktadır. Güney Kaliforniya Üniversitesi Yaratıcı Teknoloji Enstitüsü'nden, kıdemli askerlerde yaygın olan travma sonrası stres bozukluğu üzerinde çalışmıştır. VR uygulayarak stresli durumları kademeli olarak yeniden yaratmıştır. Simülasyon, travmaya neden olan tüm duyguları hastaya iade etme fikrine dayanmaktadır. Hastanın yüzleşmeye hazır olmadığı herhangi bir şeyi dışlanarak, kendini geçmiş deneyime kaptırması ve bunu kademeli olarak yapabilmesi olasılık dahilindedir (Rizzo ve Kim, 2005).

1990'lardan beri yeme bozukluklarının tedavisinde VR'nin kullanımına ilişkin ilk çalışmalar yapılmıştır (Riva, vd., 2004). Sanal dünya aracılığıyla aslında iki vücut görüntüsünü karşılaştırmak mümkündür. Hastanın vücudunun objektif ölçümünden elde edilen gerçek görüntü ve hastanın nasıl göründüğünden yola çıkarak özne tarafından algılanan görüntü karşılaştırılmaktadır (Riva, vd., 2001; Riva, vd., 2004). VR, negatif bir vücut görüntüsünü değiştirmek için olası bir araç olarak kabul edilmektedir (Riva, 2011).

Son yıllarda, ağrı için VR üzerine yapılan araştırmalarla birlikte, etkili bir nonfarmakolojik analjezik yaklaşım (MBSR meditasyonu) uygulanarak, VR'nin kronik ağrı hastaları için daha uygun olduğuna ve uzun vadede yararlı uygun bir yaklaşım sağlayabileceğine inanılmaktadır. Tong ve diğerleri (2015) tarafından bir ağrı kliniğinde (n=13) kronik ağrı tedavisinde kullanılmak üzere, biyogeribildirim sensörlerini, sürükleyici bir sanal ortamı ve stereoskopik sesi bir araya getiren farkındalık meditasyonu yoluyla hastaların dikkatlerini içe yönlendirmelerine yardımcı olmak için "Sanal Meditatif Yürüyüş" (VMW) adlı VR sistemi geliştirilmiştir. VMW, hastaların fizyolojik biyogeribildirim verileriyle eşleştirilen gerçek zamanlı sürükleyici görsel sinyaller ve sonik geri bildirim sağlayarak kronik ağrılarını yönetmelerini sağlamaktadır. VR ve MBSR meditasyonunun kronik ağrıyı yönetmede etkili olduğu kanıtlanmıştır. Benzer şekilde Gromala ve diğerlerinin (2015) VMW uygulaması yaptığı çalışmada ( $n=13), V M W ' n i n$ algılanan ağrıyı azaltmada etkili olduğu vurgulanmaktadir. 


\section{SANAL GERÇEKLİK UYGULAMALARIYLA MİNDFULNESS TEMELLI STRES AZALTMA PROGRAMININ HEMŞİRELİKTE KULLANIMI}

Son yıllarda klinik alanda VR kullanımında hem bilgi teknolojisinin kaydettiği ilerleme hem de bu teknolojinin uygulanmasındaki maliyetlerdeki düşüş için istikrarlı bir artış olmuştur (Riva, 2002). Bununla birlikte, VR cihazlarının ve yazılımlarının standartlaştırılması, yani araştırma topluluğu tarafından paylaşılabilecek standartlaştırılmış protokollerin eksikliği gibi hala birçok sınırlama vardır. Şu anda, yalnızca aşağıdaki klinik protokoller mevcuttur: yeme bozukluklarının tedavisi (Riva, vd., 2011), uçma korkusu (Rothbaum, vd., 1999; Maldonato, vd., 2018), topluluk önünde konuşma korkusu (Botella, vd., 2000), panik bozukluklar gibi (Vincelli, vd., 2011). Ayrıca, bugüne kadar, mevcut çeşitli VR sistemlerinden bazıları birlikte çalışabilir. Hemşirenin klinik becerilerinin, VR sistemlerinin klinik alanında etkin kullanım için anahtar faktör olmaya devam ettiğini ve ikame olarak değil terapi desteği olarak anlaşılacağını unutmamak gerekir. Dahası, ilişki ve iletişim ağının, sanal ortamları bireylerin, nesnelerin ve süreçlerin etkileşimde bulunduğu hasta ve terapist arasında paylaşılan dünyalara dönüştürmek için ana unsur olarak kaldığı her zaman akılda tutulmalıdır (Riva, 2005).

Hemşirelik mesleğinin, bilişsel olduğu kadar psikomotor ve tutumsal becerilerin de kazandırılması gereken bir meslek olması, eğitimde yenilikçi uygulamaların kullanılmasını zorunlu kılmaktadır (Padilha vd., 2019; Hirt ve Beer, 2020). Teknolojinin hızlı gelişimi ile birlikte hemşirelik eğitiminde VR teknolojisi kullanılarak oluşturulan sanal ortam laboratuvarlarını gündeme getirmiştir. VR teknolojisinin eğitimde kullanılmasını destekleyen pek çok çalışma mevcuttur (King vd., 2018; Padilha vd., 2019; Kardong-Edgren vd., 2019; Ayed vd., 2019; McCarthy ve Uppot, 2019; Kardong-Edgren vd., 2019; Hirt ve Beer, 2020). VR teknolojisi hemşirelik öğrencilerinin, hasta güvenliğini sağlarken temel becerilerini geliştirmelerine yardımcı olacak yenilikçi yöntemlerden biridir. VR başlığı ve oyun tabanlı öğrenme teknolojisi hemşirelik öğrencilerinde beceriyi arttırmak, kalıcılığı sağlamak için bir araç olarak kullanulabilir (Kardong-Edgren vd., 2019; McCarthy ve Uppot, 2019). Klinik alanlarda, hastaların dikkatini başka yöne çekmek, ağrının azaltılması, konforun ve rahatlığın sağlanması için kullanılan hemşirelik girişimleri arasında, son zamanlarda VR gözlükleri kullanılmaktadır. VR, teknolojinin gelişmesiyle nonfarmakolojik uygulamalar arasında kabul edilmektedir (King vd., 2018; Kardong-Edgren vd., 2019; Ayed vd., 2019).

VR teknolojileri, ruh sağlı̆̆1 müdahalelerini geniş halk kitleleri için daha erişilebilir hale getirmiştir (Hill, vd., 2017). Bu sunum platformları, farkındalık temelli müdahalelerin erişilebilirliğini geliştirmesine rağmen, araştırmalar Web ve mobil programlara bağlılığın genellikle düşük olduğunu göstermiş olup (Toivonen, vd., 2017), bu da uzun vadede ilgi çekici olmayabileceklerini düşündürmektedir (Kvillemo, vd., 2016). Bu teknolojilerin, farkındalık uygulamasıyla ilişkili bazı deneyimsel zorlukların üstesinden gelmede kullanıcılara anlamlı bir şekilde yardımcı olmasını mümkün k1lamamaktadır, bu da bağlantının kesilmesine ve hatta olumsuz etkilere katkıda bulunabilir (Blanck, vd., 2018; Anderson ve Farb, 2018). Bu konuda mindfulness temelli profesyonel uygulamalar için eğitimli hemşirelere ihtiyaç artmaktadır.

\section{SONUÇ ve ÖNERİLER}

Sonuç olarak çalışmalar, VR'ın farkındalığ 1 ve refahı iyileştirmede kullanım potansiyeli olduğunu göstermektedir. Bilgi işlemde farkındalık, sınırlı sayıda çalışma ile nispeten yeni bir araştırma alanıdır ve sanal gerçeklik oyunlarında farkındalık konusundaki literatür daha da azdır. Bu nedenle, sanal gerçeklik ve diğer dijital uygulamalarda farkındalık ile ilgili süreçleri anlamak için daha fazla araştırmaya ihtiyaç vardır. Farkındalıkla ilgili sanal gerçeklik oyunlarında neurofeedback'in kullanılması gelecekteki uygulamalar için umut verici görünüyor. VR gibi yeni teknolojilerin hemşirelik uygulamaları ve hemşirelik eğitiminde benimsenmesinin artmasının, hemşire-hasta ilişkilerini olumlu yönde değiştireceği, potansiyel faydalar sunacağı düşünülmektedir. Günümüzde teknolojik gelişmelerle birlikte sağlık alanında VR teknolojilerinin kullanımının önemi de giderek artmaktadır. Bu bağlamda hemşirelerin ve tüm sağlık profesyonellerinin teknolojiye ayak uydurmaları kaçınılmazdır. Koruyucu, tedavi edici ve rehabilite edici sağlık hizmetlerinde VR uygulamalarından yararlanılmasının önemli ve gerekli olduğu düşünülmektedir. 


\section{KAYNAKÇA}

ALDAO, A., NOLEN-HOEKSEMA, S., \& SCHWEIZER, S. (2010). Emotion-regulation strategies across psychopathology: A meta-analytic review. Clinical psychology review, 30(2), 217-237.

AMORES, J., BENAVIDES, X., \& MAES, P. (2016, May). Psychicvr: Increasing mindfulness by using virtual reality and brain computer interfaces. In Proceedings of the 2016 CHI Conference Extended Abstracts on Human Factors in Computing Systems (pp. 2-2).

ANDERSON, T., \& FARB, N. A. (2018). Personalising practice using preferences for meditation anchor modality. Frontiers in psychology, 9, 2521.

ASATI, M., \& MIYACHI, T. (2019). A Short Virtual Reality Mindfulness Meditation Training For Regaining Sustained Attention. arXiv preprint arXiv:1907.04487.

AYED, I., GHAZEL, A., JAUME-İ-CAPO, A., MOYÀ-ALCOVER, G., VARONA, J., \& MARTÍNEZ-BUESO, P. (2019). Vision-based serious games and virtual reality systems for motor rehabilitation: A review geared toward a research methodology. International journal of medical informatics, 131, 103909.

BAER, R. A., SMITH, G. T., LYKINS, E., BUTTON, D., KRIETEMEYER, J., SAUER, S., ... \& WILLIAMS, J. M. G. (2008). Construct validity of the five facet mindfulness questionnaire in meditating and nonmeditating samples. Assessment, 15(3), 329-342.

BIRNIE, K., SPECA, M., \& CARLSON, L. E. (2010). Exploring self-compassion and empathy in the context of mindfulness-based stress reduction (MBSR). Stress and Health, 26(5), 359-371.

BISHOP, S. R., LAU, M., SHAPIRO, S., CARLSON, L., ANDERSON, N. D., CARMODY, J., ... \& DEVINS, G. (2004). Mindfulness: A proposed operational definition. Clinical psychology: Science and practice, 11(3), 230241.

BLANCK, P., PERLETH, S., HEIDENREICH, T., KRÖGER, P., DITZEN, B., BENTS, H., \& MANDER, J. (2018). Effects of mindfulness exercises as stand-alone intervention on symptoms of anxiety and depression: Systematic review and meta-analysis. Behaviour Research and Therapy, 102, 25-35. BOTELla, C., BANOS, R., GUILlÉN, V., PERPIÑÁ, C., ALCAÑIZ, M., $\&$ PONS, A. (2000). Telepsychology: Public speaking fear treatment on the internet. CyberPsychology \& Behavior, 3(6), 959-968.

BRUGGEMAN, K. J., \& WURSTER, S. W. (2018). The Hiatus system: virtual healing spaces: low dose mindfulness based stress reduction virtual reality application. In ACM SIGGRAPH 2018 Appy Hour (pp. 1-2).

CAMPOS, D., CEBOLLA, A., QUERO, S., BRETÓN-LÓPEZ, J., BOTELLA, C., SOLER, J., ... \& BAÑOS, R. M. (2016). Meditation and happiness: Mindfulness and self-compassion may mediate the meditation-happiness relationship. Personality and individual differences, 93, 80-85.

CHAMBERS, R., LO, B. C. Y., \& ALLEN, N. B. (2008). The impact of intensive mindfulness training on attentional control, cognitive style, and affect. Cognitive therapy and research, 32(3), 303-322.

CHANDRASIRI, A., COLLETT, J., FASSBENDER, E., \& DE FOE, A. (2020). A virtual reality approach to mindfulness skills training. Virtual Reality, 24(1), 143-149.

CHOO, A., \& MAY, A. (2014, October). Virtual mindfulness meditation: Virtual reality and electroencephalography for health gamification. In 2014 IEEE Games Media Entertainment (pp. 1-3). IEEE.

CIKAJLO, I., STABA, U. C., VRHOVAC, S., LARKIN, F., \& RODDY, M. (2017). A cloud-based virtual reality app for a novel telemindfulness service: rationale, design and feasibility evaluation. JMIR research protocols, 6(6), e6849.

COLlins, E., COX, A., WILCOCK, C., \& SETHU-JONES, G. (2019). Digital games and mindfulness apps: comparison of effects on post work recovery. JMIR mental health, 6(7), e12853.

COSTA, R. T. D., CARVAlHO, M. R. D., RIBEIRO, P., \& NARDI, A. E. (2018). Virtual reality exposure therapy for fear of driving: analysis of clinical characteristics, physiological response, and sense of presence. Revista brasileira de psiquiatria, (AHEAD), 0-0.

CRESCENTINI, C., ChITTARO, L., CAPURSO, V., SIONI, R., \&
FABBRO, F. (2016). Psychological and physiological responses to stressful situations in immersive virtual reality: Differences between users who practice mindfulness meditation and controls. Computers in Human Behavior, 59, 304-316.

DAMEN, K. H., \& VAN DER SPEK, E. D. (2018, September). Virtual reality as e-mental health to support starting with mindfulness-based cognitive therapy. In International Conference on Entertainment Computing (pp. 241247). Springer, Cham.

DIEMER, J., ALPERS, G. W., PEPERKORN, H. M., SHIBAN, Y., \& MÜHLBERGER, A. (2015). The impact of perception and presence on emotional reactions: a review of research in virtual reality. Frontiers in psychology, 6 , 26.

DIEMER, J., MÜHLBERGER, A., PAULI, P., \& ZWANZGER, P. (2014). Virtual reality exposure in anxiety disorders: Impact on psychophysiological reactivity. The World Journal of Biological Psychiatry, 15(6), 427-442.

ERISMAN, S. M., \& ROEMER, L. (2010). A preliminary investigation of the effects of experimentally induced mindfulness on emotional responding to film clips. Emotion, 10(1), 72.

FLORES, A., LINEHAN, M. M., TODD, S. R., \& HOFFMAN, H. G. (2018). The use of virtual reality to facilitate mindfulness skills training in dialectical behavioral therapy for spinal cord injury: a case study. Frontiers in psycho$\log y, 9,531$.

GIRARDEAU, J. C., BLONDÉ, P., MAKOWSKI, D., ABRAM, M., PIOLINO, P., \& SPERDUTI, M. (2020). The impact of state and dispositional mindfulness on prospective memory: a virtual reality study. Consciousness and cognition, 81, 102920.

GOMEZ, J., HOFFMAN, H. G., BISTRICKY, S. L., GONZALEZ, M., ROSENBERG, L., SAMPAIO, M., ... \& LINEHAN, M. M. (2017). The use of Virtual Reality Facilitates Dialectical Behavior Therapy ${ }^{\circledR}$ "Observing Sounds and Visuals" mindfulness skills training exercises for a Latino patient with severe burns: a case study. Frontiers in psychology, 8, 1611.

GROMALA, D., SONG, M., YIM, J. D., FOX, T., BARNES, S. J., NAZEMI, M., ... \& SQUIRE, P. (2011). Immersive VR: A non-pharmacological analgesic for chronic pain?. In CHI'11 Extended Abstracts on Human Factors in Computing Systems (pp. 1171-1176).

GROMALA, D., TONG, X., CHOO, A., KARAMNEJAD, M., \& SHAW, C. D. (2015, April). The virtual meditative walk: virtual reality therapy for chronic pain management. In Proceedings of the 33rd Annual ACM Conference on Human Factors in Computing Systems (pp. 521-524).

GROSSMAN, P., NIEMANN, L., SCHMIDT, S., \& WALACH, H. (2004). Mindfulness-based stress reduction and health benefits: A meta-analysis. Journal of psychosomatic research, 57(1), 35-43.

HAYES, S. C., STROSAHL, K. D., \& WILSON, K. G. (2009). Acceptance and commitment therapy. Washington, DC: American Psychological Association.

HILL, C., MARTIN, J. L., THOMSON, S., SCOTT-RAM, N., PENFOLD, H., \& CRESWELL, C. (2017). Navigating the challenges of digital health innovation: considerations and solutions in developing online and smartphone-application-based interventions for mental health disorders. The British Journal of Psychiatry, 211(2), 65-69.

HIRT, J., \& BEER, T. (2020). Use and impact of virtual reality simulation in dementia care education: A scoping review. Nurse education today, 84, 104207.

IRVING, J. A., DOBKIN, P. L., \& PARK, J. (2009). Cultivating mindfulness in health care professionals: A review of empirical studies of mindfulness-based stress reduction (MBSR). Complementary therapies in clinical practice, 15(2), 61-66.

JHA, A. P., STANLEY, E. A., KIYONAGA, A., WONG, L., \& GELFAND, L. (2010). Examining the protective effects of mindfulness training on working memory capacity and affective experience. Emotion, 10(1), 54.

KABAT-ZINN, J., DE TORRIJOS, F., SKILLINGS, A. H., BLACKER, M., MUMFORD, G. T., ALVARES, D. L., ... \& ROSAL, M. C. (2016). Delivery and Effectiveness of a Dual Language (English/Spanish) Mindfulness-Based Stress Reduction (MBSR): Program in the Inner City-A Seven-Year Experience: 1992-1999. Mindfulness \& Compassion, 1(1), 2-13. 
KABAT-ZINN, J. (1982). An outpatient program in behavioral medicine for chronic pain patients based on the practice of mindfulness meditation Theoretical considerations and preliminary results. General hospital psychiatry, 4(1), 33-47.

KABAT-ZINN, J. (1990). Full catastrophe living: The program of the stress reduction clinic at the University of Massachusetts Medical Center

KABAT-ZINN, J. (2011). Some reflections on the origins of MBSR, skillful means, and the trouble with maps. Contemporary Buddhism, 12(1), 281-306. KARDONG-EDGREN, S. S., FARRA, S. L., ALINIER, G., \& YOUNG, H. M. (2019). A call to unify definitions of virtual reality. Clinical Simulation in Nursing, 31, 28-34

KHOURY, B., LECOMTE, T., GAUDİANO, B. A., \& PAQUIN, K. (2013) Mindfulness interventions for psychosis: a meta-analysis. Schizophrenia research, 150(1), 176-184

KING, D., TEE, S., FALCONER, L., ANGELL, C., HOLLEY, D., \& MILLS, A. (2018). Virtual health education: Scaling practice to transform student learning: Using virtual reality learning environments in healthcare education to bridge the theory/practice gap and improve patient safety. Nurse Educ Today, 71, 7-9.

KOSUNEN, I., SALMINEN, M., JÄRVELÄ, S., RUONALA, A., RAVAJA, N., \& JACUCCI, G. (2016, March). RelaWorld: neuroadaptive and immersive virtual reality meditation system. In Proceedings of the 21st International Conference on Intelligent User Interfaces (pp. 208-217).

KUYKEN, W., WATKINS, E., HOLDEN, E., WHITE, K., TAYLOR, R. S., BYFORD, S., ... \& DALGLEISH, T. (2010). How does mindfulness-based cognitive therapy work?. Behaviour research and therapy, 48(11), 11051112 .

KVILLEMO, P., BRANDBERG, Y., \& BRÄNSTRÖM, R. (2016). Feasibility and outcomes of an internet-based mindfulness training program: a pilot randomized controlled trial. JMIR mental health, 3(3), e33.

LINDSAY, E. K., \& CRESWELL, J. D. (2017). Mechanisms of mindfulness training: Monitor and Acceptance Theory (MAT). Clinical psychology review, 51, 48-59.

LINEHAN, M. (2015). DBT skills training manual. Dialectical behavior therapy skills training manual (Second edi). Guilford Press, New York.

LITTLE, D. (2016). Supporting or inhibiting motivations to meditate? A semi-structured interview study of the user experience of mobile mindfulness apps. UCL Interaction Centre.

LYMEUS, F., LUNDGREN, T., \& HARTIG, T. (2017). Attentional effort of beginning mindfulness training is offset with practice directed toward images of natural scenery. Environment and Behavior, 49(5), 536-559.

LUTZ, A., SLAGTER, H. A., DUNNE, J. D., \& DAVIDSON, R. J. (2008). Attention regulation and monitoring in meditation. Trends in cognitive sciences, 12(4), 163-169.

MALDONATO, M. N., SPERANDEO, R., DELL'ORCO, S., IENNACO, D., CERRONI, F., ROMANO, P., ... \& MAROTTA, R. (2018). Mind brain and altered states of consciousness. Acta Medica Mediterranea, 34, 357.

MCCARTHY, C. J., \& UPPOT, R. N. (2019). Advances in virtual and augmented reality - exploring the role in health-care education. Journal of Radiology Nursing, 38(2), 104-105.

MILOFF, A., MARKLUND, A., \& CARLBRING, P. (2015). The challenger app for social anxiety disorder: New advances in mobile psychological treatment. Internet Interventions, 2(4), 382-391.

MONTERO-MARIN, J., PUEBLA-GUEDEA, M., HERRERA-MERCADAL, P., CEBOLLA, A., SOLER, J., DEMARZO, M., ... \& GARCÍA-CAMPAYO, J. (2016). Psychological effects of a 1-month meditation retreat on experienced meditators: the role of non-attachment. Frontiers in Psycho$\log y, 7,1935$.

MONTERO-MARIN, J., TOPS, M., MANZANERA, R., PIVA DEMARZO, M. M., ALVAREZ DE MON, M., \& GARCÍA-CAMPAYO, J. (2015). Mindfulness, resilience, and burnout subtypes in primary care physicians: the possible mediating role of positive and negative affect. Frontiers in psycho$\log y, 6,1895$.

NAVARRO-HARO, M. V., LÓPEZ-DEL-HOYO, Y., CAMPOS, D., LINE-
HAN, M. M., HOFFMAN, H. G., GARCÍA-PALACIOS, A., .. \& GARCÍA-CAMPAYO, J. (2017). Meditation experts try Virtual Reality Mindfulness: A pilot study evaluation of the feasibility and acceptability of Virtual Reality to facilitate mindfulness practice in people attending a Mindfulness conference. PloS one, 12(11), e0187777.

NAVARRO-HARO, M. V., MODREGO-ALARCÓN, M., HOFFMAN, H. G., LÓPEZ-MONTOYO, A., NAVARRO-GIL, M., MONTERO-MARIN, J., ... \& GARCÍA-CAMPAYO, J. (2019). Evaluation of a mindfulness-based intervention with and without virtual reality dialectical behavior therapy ${ }^{\circledR}$ mindfulness skills training for the treatment of generalized anxiety disorder in primary care: A pilot study. Frontiers in psychology, 10, 55.

NEFF, K. (2003). Self-compassion: An alternative conceptualization of a healthy attitude toward oneself. Self and identity, 2(2), 85-101.

PADILHA, J. M., MACHADO, P. P., RIBBEIRO, A., RAMOS, J., \& COSTA, P. (2019). Clinical virtual simulation in nursing education: randomized controlled trial. Journal of medical Internet research, 21(3), e11529.

RIVA, G., BACCHETTA, M., CESA, G., CONTI, S., \& MOLINARI, E. (2004). The use of VR in the treatment of eating disorders

RIVA, G., BACCHETTA, M., CESA, G., CONTI, S., \& MOLINARI, E. (2001). Virtual reality and telemedicine based experiential cognitive therapy: Rationale and clinical protocol.

RIVA, G. (2011). The key to unlocking the virtual body: virtual reality in the treatment of obesity and eating disorders. Journal of diabetes science and technology, 5(2), 283-292.

RIVA, G. (2002). Virtual reality for health care: the status of research. $C y$ berpsychology \& Behavior, 5(3), 219-225.

RIVA, G. (2005). Virtual reality in psychotherapy. Cyberpsychology \& behavior, 8(3), 220-230.

RIZZO, A. S., \& KIM, G. J. (2005). A SWOT analysis of the field of virtual reality rehabilitation and therapy. Presence: Teleoperators \& Virtual Environments, 14(2), 119-146

ROQUET, D. C., \& SAS, C. (2018, April). Evaluating mindfulness meditation apps. In Extended Abstracts of the 2018 CHI Conference on Human Factors in Computing Systems (pp. 1-6)

ROTHBAUM, B. O., HODGES, L., \& SMITH, S. (1999). Virtual reality exposure therapy abbreviated treatment manual: Fear of flying application. Cognitive and Behavioral Practice, 6(3), 234-244.

SEABROOK, E., KELLY, R., FOLEY, F., THEILER, S., THOMAS, N., WADLEY, G., \& NEDELJKOVIC, M. (2020). Understanding how virtual reality can support mindfulness practice: Mixed methods study. Journal of medical Internet research, 22(3), e16106.

SEGAL, Z. V., WILLIAMS, J. M. G., TEASDALE, J. D. (2002). Mindfulness-based cognitive therapy for depression: A new approach to preventing relapse. Guilford Press, New York.

SEGAL, Z. V., WILliAMS, J. M. G., TEASDALE, J. D. (2002). Mindfulness-based cognitive therapyfor depression: A new approach to preventing relapse. Guilford Press, New York.

SEVILLA-LLEWELLYN-JONES, J., SANTESTEBAN-ECHARRI, O., PRYOR, I., MCGORRY, P., \& ALVAREZ-JIMENEZ, M. (2018). Web-based mindfulness interventions for mental health treatment: systematic review and meta-analysis. JMIR mental health, 5(3), e10278.

SHAW, C., GROMALA, D., \& SONG, M. (2011). The meditation chamber: towards self-modulation. In Metaplasticity in virtual worlds: Aesthetics and semantic concepts (pp. 121-133). IGI Global.

SLATER, M. (2003). A note on presence terminology [online] http://s3.amazonaws.com/publicationslist.org/data/melslater/ref201/a $\% 20$ note $\% 20$ on\%20presence\%20terminology.pdf [Erişim Tarihi: 14 Ocak 2021]

SLATER, M. (2014). Grand challenges in virtual environments. Frontiers in Robotics and AI, 1,3 .

SLATER, M. (1999). Measuring presence: A response to the Witmer and Singer presence questionnaire. Presence, 8(5), 560-565.

STEUER, J. (1992). Defining virtual reality: Dimensions determining telepresence. Journal of communication, 42(4), 73-93.

TOIVONEN, K. I., ZERNICKE, K., \& CARLSON, L. E. (2017). Web-based 
mindfulness interventions for people with physical health conditions: systematic review. Journal of medical Internet research, 19(8), e303.

TONG, X., GROMALA, D., CHOO, A., AMIN, A., \& SHAW, C. (2015, August). The virtual meditative walk: an immersive virtual environment for pain self-modulation through mindfulness-based stress reduction meditation. In International Conference on Virtual, Augmented and Mixed Reality (pp. 388-397). Springer, Cham.

VINCELLI, F., ANOLLI, L., BOUCHARD, S., WIEDERHOLD, B. K., ZURLONI, V., \& RIVA, G. (2003). Experiential cognitive therapy in the treatment of panic disorders with agoraphobia: a controlled study. CyberPsychology \& Behavior, 6(3), 321-328.

WAHBEH, H., \& OKEN, B. S. (2016). Internet mindfulness meditation intervention for the general public: pilot randomized controlled trial. JMIR mental health, 3(3), e37.

WITMER, B. G., \& SINGER, M. J. (1998). Measuring presence in virtual environments: A presence questionnaire. Presence, 7(3), 225-240. 